\title{
LETTERS
}

\section{Weighing of biomolecules, single cells and single nanoparticles in fluid}

\author{
Thomas P. Burg ${ }^{1 \star}$, Michel Godin ${ }^{1 \star}$, Scott M. Knudsen ${ }^{1}$, Wenjiang Shen ${ }^{3}$, Greg Carlson ${ }^{3}$, John S. Foster ${ }^{3}$, \\ Ken Babcock ${ }^{3,4} \&$ Scott R. Manalis ${ }^{1,2}$
}

Nanomechanical resonators enable the measurement of mass with extraordinary sensitivity ${ }^{1-7}$. Previously, samples as light as 7 zeptograms $\left(1 \mathrm{zg}=10^{-21} \mathrm{~g}\right)$ have been weighed in vacuum, and protonlevel resolution seems to be within reach $^{8}$. Resolving small mass changes requires the resonator to be light and to ring at a very pure tone-that is, with a high quality factor'. In solution, viscosity severely degrades both of these characteristics, thus preventing many applications in nanotechnology and the life sciences where fluid is required ${ }^{10}$. Although the resonant structure can be designed to minimize viscous loss, resolution is still substantially degraded when compared to measurements made in air or vacuum $^{11-14}$. An entirely different approach eliminates viscous damping by placing the solution inside a hollow resonator that is surrounded by vacuum ${ }^{15,16}$. Here we demonstrate that suspended microchannel resonators can weigh single nanoparticles, single bacterial cells and sub-monolayers of adsorbed proteins in water with sub-femtogram resolution $(1 \mathrm{~Hz}$ bandwidth). Central to these results is our observation that viscous loss due to the fluid is negligible compared to the intrinsic damping of our silicon crystal resonator. The combination of the low resonator mass $(100 \mathrm{ng})$ and high quality factor $(15,000)$ enables an improvement in mass resolution of six orders of magnitude over a high-end commercial quartz crystal microbalance ${ }^{17}$. This gives access to intriguing applications, such as mass-based flow cytometry, the direct detection of pathogens, or the non-optical sizing and mass density measurement of colloidal particles.

The resonance frequency of a suspended microfluidic channel with micrometre-thin walls and a comparably thin fluid layer is highly sensitive to the presence of molecules or particles whose mass density differs from that of the solution (Fig. 1). This enables experiments that extend conceptually beyond the measurement of bulk fluid density, which has been demonstrated previously ${ }^{18,19}$. As the ratio of surface area to volume of our suspended microchannel is very large $\left(10^{4} \mathrm{~cm}^{-1}\right)$, surface adsorption is an effective mechanism for biomolecular mass sensing (Fig. 1b) $)^{15}$.

In another measurement mode (Fig. 1c), particles suspended in the solution flow through the resonator, and the resulting frequency shift depends on the mass and position of the particles. For dilute suspensions, this measurement yields a series of well separated peaks whose heights are directly proportional to the mass excess of each particle in solution.

Changes in mass inside the channel translate into shifts in the resonance frequency, $f$, according to ${ }^{20}$ :

$$
f=\frac{1}{2 \pi} \sqrt{\frac{k}{m^{*}+\alpha \Delta m}}
$$

The spring constant of the resonator is denoted by $k, m^{*}$ is the

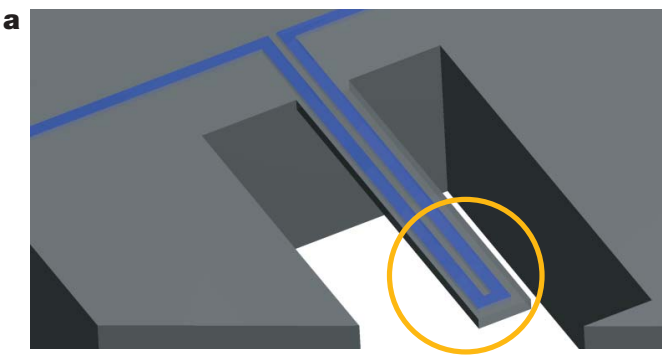

b

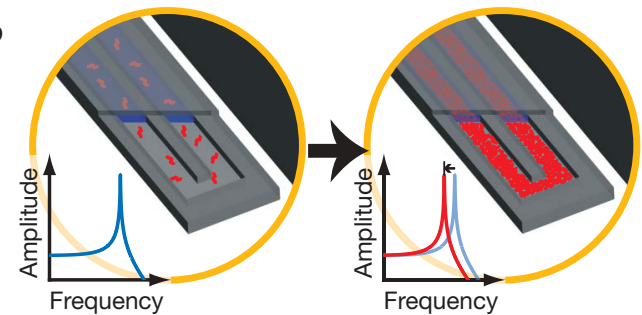

c

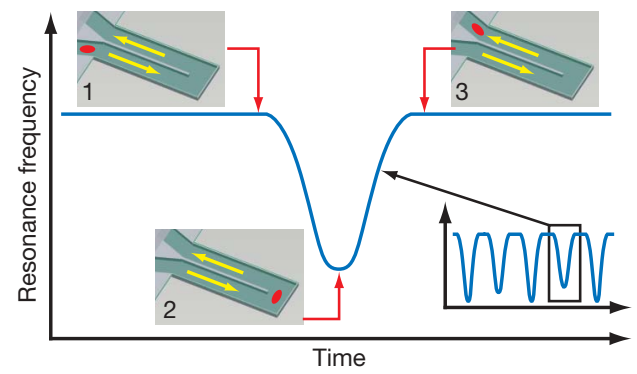

Figure 1 | Illustration of two mass measurement modes enabled by a fluidfilled microcantilever. a, A suspended microchannel translates mass changes into changes in resonance frequency. Fluid continuously flows through the channel and delivers biomolecules, cells or synthetic particles. Sub-femtogram mass resolution is attained by shrinking the wall and fluid layer thickness to the micrometre scale and by packaging the cantilever under high vacuum. $\mathbf{b}$, While bound and unbound molecules both increase the mass of the channel, species that bind to the channel wall accumulate inside the device, and, as a result, their number can greatly exceed the number of free molecules in solution. This enables specific detection by way of immobilized receptors. c, In another measurement mode, particles flow through the cantilever without binding to the surface, and the observed signal depends on the position of particles along the channel (insets 1-3). The exact mass excess of a particle can be quantified by the peak frequency shift induced at the apex.

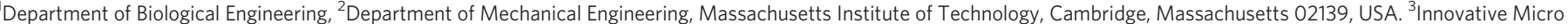
Technology, Santa Barbara, California 93117, USA. ${ }^{4}$ Affinity Biosensors, Santa Barbara, California 93117, USA.

*These authors contributed equally to this work. 
effective mass, and $\alpha$ is a numerical constant that depends on the geometric localization of the added mass $\Delta m$. For our cantilevershaped resonators, $\alpha$ takes on a value of $\sim 0.24$ for changes in solution density and for uniform adsorption of molecules to the surface, and $\alpha \approx 1$ when a particle in transit is positioned at the apex.

Our fabrication process is based on the creation of buried channels in silicon-on-insulator wafers, followed by wafer thinning and dry etching to form suspended microchannels with walls $2-3 \mu \mathrm{m}$ thick and a $3 \mu \mathrm{m}$ fluid layer. Two hundred devices are fabricated and vacuum-packaged on a six-inch wafer, with yields exceeding $80 \%$. A 'getter' layer prevents slow degradation of the on-chip vacuum due to outgassing. Integrated under each cantilever is an electrostatic drive electrode (Fig. 2a), and the cantilever vibration is detected optically. Further detail regarding the device fabrication is provided in Supplementary Information.

Dry resonators possess quality factors up to 15,000 and, as shown by the frequency response plot in Fig. $2 b$, no increase in damping occurs after filling the device with water. In comparison, conventional microresonators submersed in fluid have quality factors of up to $\sim 150$ (refs $11-14$ ). Owing to the high quality factor, a gain controlled oscillator circuit with our device as the frequency determining element exhibits a frequency stability of 10 p.p.b. (parts per billion) in a $1 \mathrm{~Hz}$ bandwidth, even when the suspended microchannel is subjected to continuous flow. As the oscillator continuously tracks the resonance frequency, the dynamic range exceeds seven orders of

a

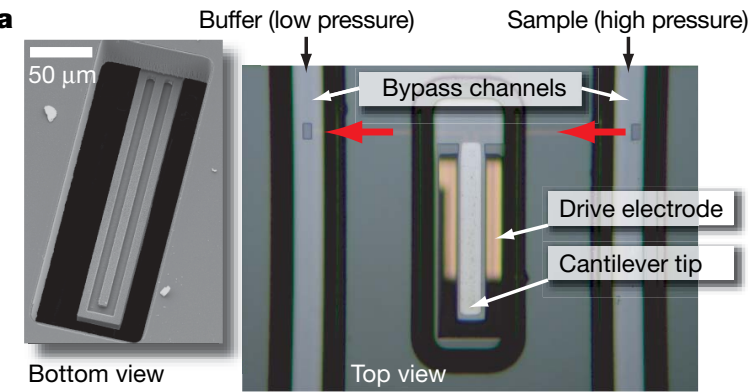

b

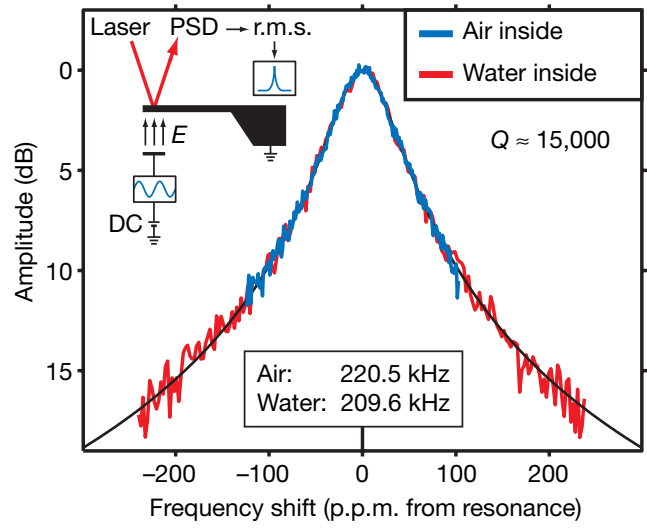

Figure 2 | Micrographs and frequency response of a suspended microchannel resonator. a, The $200 \times 33 \times 7 \mu \mathrm{m}$

(length $\times$ width $\times$ thickness) microcantilever containing a $3 \times 8 \mu \mathrm{m}$ (height $\times$ width) channel is suspended in a vacuum cavity (optical micrograph, right). Microfluidic bypass channels $(30 \times 100 \mu \mathrm{m}$, height $\times$ width) are connected to the inlet and the outlet of the suspended channel, and enable the quick exchange of samples by pressure driven flow (red arrows). The electron micrograph (left) shows a bottom view of a cantilever that has been intentionally etched open to visualize the fluidic conduit inside. b, Frequency response plots of a cantilever before (blue) and after (red) filling with water reveal different resonance frequencies but indistinguishable quality factors. To measure the frequency response, we monitored the vibration amplitude with a laser and a position sensitive photodetector (PSD) while the cantilever was being driven electrostatically at different frequencies (inset; $E$ denotes the electric field, and 'DC' represents a bias voltage of $\sim 60 \mathrm{~V}$ ). magnitude for short measurements (of the order of seconds with a $1 \mathrm{~Hz}$ bandwidth). Over extended periods of time, drift becomes the limiting factor, and at higher bandwidths frequency resolution is limited by phase noise.

Perhaps the most widely used mass-sensing method today is the quartz crystal microbalance, with a noise floor in solution of $\sim 1 \mathrm{ng} \mathrm{cm}^{-2}$ and a total mass resolution of $\sim 1 \mathrm{ng}$ (ref. 17). Microcantilevers in solution have been used to resolve mass changes of $\sim 1 \mu \mathrm{g} \mathrm{cm}^{-2}(\sim 1 \mathrm{ng} \text { total mass })^{13}$ and other micromachined resonators optimized for fluid operation have achieved $10 \mathrm{ng} \mathrm{cm}^{-2}$ (ref. 12). In the context of biomolecular detection, surface plasmon resonance (SPR) is widely regarded as the 'gold standard'. Although the detection in SPR is based on refractive index, the signal is approximately proportional to mass, and laboratory instruments attain a resolution equivalent to $\sim 0.05 \mathrm{ng} \mathrm{cm}^{-2}(\sim 1 \mathrm{pg} \text { total mass })^{21}$. Using the suspended microchannel resonator, mass that is evenly adsorbed to the inner surfaces can be resolved to $0.01 \mathrm{ng} \mathrm{cm}^{-2}$ ( $1 \mathrm{fg}$ total). A unique aspect of our device is the ability to measure the mass of objects that are not attached to the surface. Sensitivity is highest near the cantilever tip, enabling the measurement of particle masses with a precision of 300 attograms $\left(1 \mathrm{ag}=10^{-18} \mathrm{~g}\right)$. If the frequency measurement is refined to approach the thermomechanical noise floor of our device, it should be possible to resolve mass changes as low as $1 \mathrm{ag}$, and shrinking the wall thickness and/or channel size to sub-micrometre dimensions could further improve sensitivity. A more detailed discussion of mass sensing with suspended microchannel resonators in relationship to other methods is provided in Supplementary Information.

To demonstrate the surface-based detection of proteins, we measured the binding of goat anti-mouse immunoglobulin- $\gamma$ (IgG) molecules to anti-goat IgG antibodies that were immobilized on the channel walls (Fig. 3). Biotinylated antibodies were linked to the surface via a layer of Neutravidin bound to poly(ethyleneglycol)biotin grafted poly-L-lysine (PLL-PEG-biotin). Before adsorbing the PLL-PEG-biotin, the surface was cleaned with a mixture of acetic acid and hydrogen peroxide (1:1). This mixture or, alternatively, sulphuric acid and hydrogen peroxide ('piranha solution') may be repeatedly injected into the all-glass and silicon device, thus enabling cleaning and multiple re-use of the chips.

The exact mass of the different layers can be quantified by the difference in resonance frequency before and after each injection, as shown in Fig. 3a. Directly before injections, we filled the inlet bypass with $14 \mu \mathrm{l}$ of sample at low pressure while the outlet bypass and the resonator were continuously rinsed with phosphate buffered saline (PBS) (Fig. 3a left inset). Pressure on the inlet bypass was subsequently turned on, forcing the sample through the resonator at a flow rate of $\sim 100 \mathrm{nl} \mathrm{min}^{-1}$ (Fig. 3a right inset). This method of sample introduction ensures a sharp concentration profile and minimizes reagent consumption.

We injected goat anti-mouse IgG at concentrations ranging from $0.7 \mathrm{nM}\left(100 \mathrm{ng} \mathrm{ml}^{-1}\right)$ to $0.7 \mu \mathrm{M}\left(100 \mu \mathrm{g} \mathrm{ml}^{-1}\right)$ into the functionalized resonator, and observed the binding in real time by monitoring resonance frequency (Fig. 3b). All samples were prepared in PBS containing $0.01 \%$ Tween 20 and $1 \mathrm{mg} \mathrm{ml}^{-1}$ bovine serum albumin (BSA) as a carrier protein. The signal caused by the solution density difference between the sample and the running buffer was subtracted off-line, as detailed in Supplementary Information.

Appropriate surface functionalization is crucial for biomolecular recognition with high specificity and sensitivity, and a number of specialized coatings that are compatible with silicon substrates have been developed for this purpose ${ }^{22}$. Assuming monolayer coverage (1-2 pmol $\mathrm{cm}^{-2}$ ) of active antibodies with a dissociation constant of $1 \mathrm{nM}$, we expect an ultimate limit of detection of the order of $1 \mathrm{pM}$ for a $30 \mathrm{kDa}$ analyte. In comparison, enzyme-linked immunosorbent assays (ELISA) have detection limits down to $0.1 \mathrm{pM}$. However, ELISA assays do not provide a real-time quantitative readout of antigen binding. An additional comparison can be made to microcantilever surface stress 
measurements, which have achieved detection limits of $1 \mathrm{pM}$ to $1 \mathrm{nM}$ for protein binding ${ }^{23,24}$. However, owing to the complex dependence of surface stress on molecular binding, sensitivity is highly assay dependent.

The limit of detection by mass could be improved through mass enhancement by gold labelled secondary antibodies. For instance, the binding of a single $100 \mathrm{~nm}$ Au nanoparticle near the apex of the cantilever can clearly be resolved. Although the advantage of labelfree detection is lost in this approach, mass-labelling would enable non-optical detection in a continuous-flow microfluidic device with femtomolar sensitivity. In addition, differential sensing and temperature control can be implemented to improve the detection of small signals in the presence of thermal drift, non-specific binding, or bulk density differences.

In applications where large fluid volumes need to be processed, the utility of our approach is limited by the high flow resistance of the
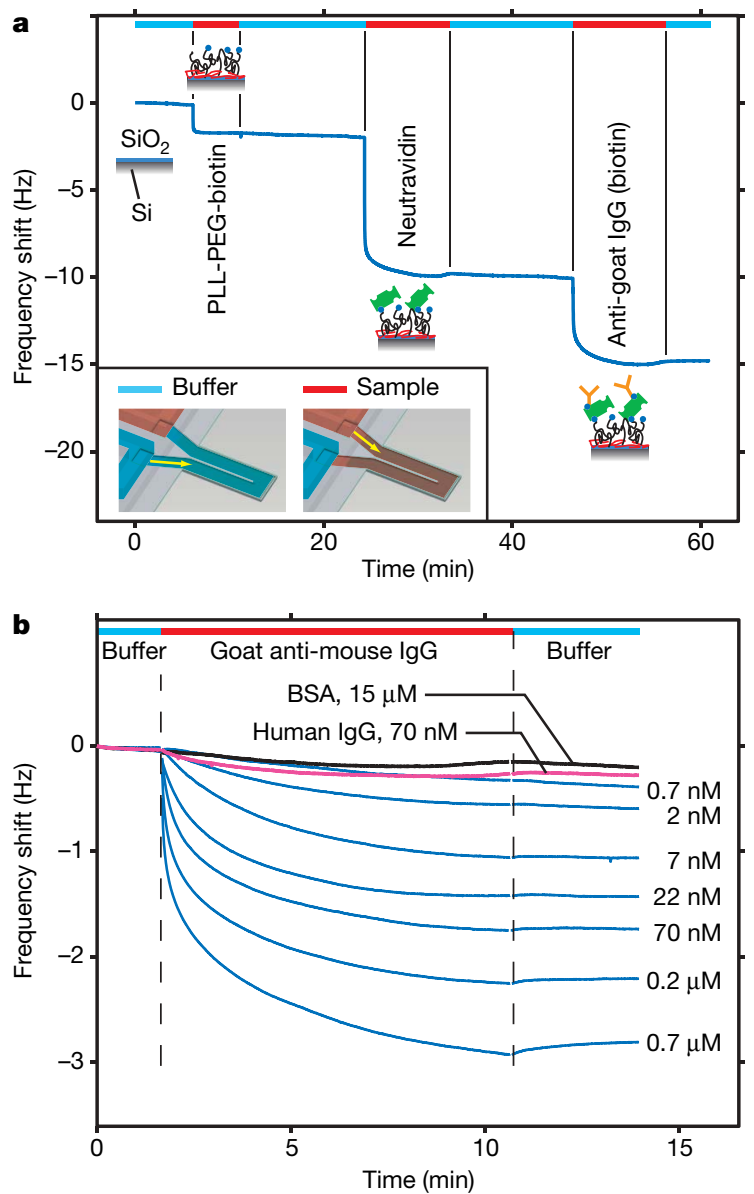

Figure 3 | Resonance frequency shifts caused by accumulation of proteins inside the cantilever. a, Antibodies to goat IgG are immobilized on the native silicon dioxide surface of the suspended microchannel resonator in three steps, as illustrated by the pictograms along the frequency trace: electrostatic adsorption of poly(ethyleneglycol)-biotin grafted poly-L-lysine (PLL-PEG-biotin, $\left.1 \mathrm{mg} \mathrm{ml}^{-1}\right)$, binding of Neutravidin $\left(0.5 \mathrm{mg} \mathrm{ml}^{-1}\right)$, and attachment of biotinylated antibodies $\left(0.5 \mathrm{mg} \mathrm{m}^{-1}\right)$ to the Neutravidin. The mass increase at each step can be followed in real time

$\left(1 \mathrm{~Hz} \approx 300 \mathrm{fmol} \mathrm{cm}^{-2}\right.$ for $150 \mathrm{kDa}$ IgG molecules). One bypass channel was continuously rinsed with phosphate buffered saline (PBS) while the other bypass contained the sample. During the injection interval (red), the bypass holding the sample was pressurized, and during rinse cycles (blue), the pressure difference was reversed, as shown in the inset at the bottom of the panel. b, Goat anti-mouse IgG was injected at concentrations from $0.7 \mathrm{nM}$ to $0.7 \mu \mathrm{M}$ (blue traces). Between measurements, the surface was regenerated by injecting $200 \mathrm{mM}$ glycine ( $\mathrm{HCl}$; $\mathrm{pH} 2.5$ ), which dissociated the analyte while preserving the activity of the antibodies. Control injections with no IgG (black) or human IgG (red) showed very low levels of non-specific binding.

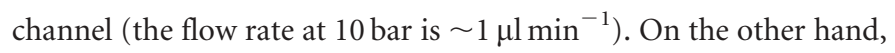
the suspended microchannel resonator enables the analysis of precious samples with minimal reagent consumption: at a flow rate of $100 \mathrm{nl} \mathrm{min}^{-1}$, the $10 \mathrm{pl}$ detector volume is swept more than 100 times per second while the large surface-to-volume ratio of the thin channel provides for a high capture efficiency.

To illustrate the flow-through detection scheme, we measured the mass of bacteria and synthetic micro- and nanoparticles in transit through the suspended microchannel. Carefully matching the pressure at the inlet and the outlet of the resonator enabled us to reduce the flow rate to less than $10 \mathrm{pls}^{-1}$. A low flow rate enables higherresolution frequency measurements by increasing the transit time of the particle through the device. Several hundred particles can be weighed individually in a few minutes, producing a histogram of particle masses (Fig. 4a). One element of uncertainty is the exact lateral position of the particles at the time they pass the cantilever tip: particles travelling along the outer sidewall of the 8 - $\mu \mathrm{m}$-wide channel induce an $8 \%$ larger frequency shift than particles following the centreline of the channel. This variability could be reduced to below $1 \%$ by hydrodynamic focusing or by using a different resonator design, thereby enabling precise weighing — or, if the density is known, sizing - of sub-micrometre particles. In cases where the
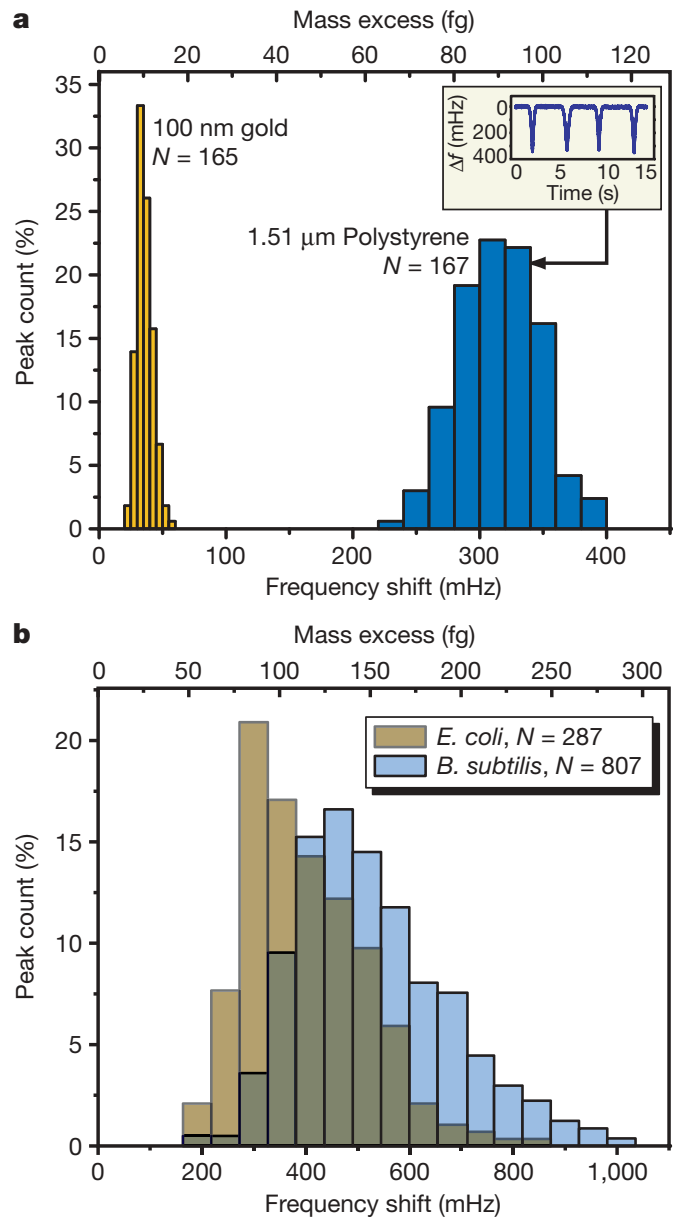

Figure 4 | Histograms of peak frequency shifts caused by particles and bacteria flowing through the resonator. a, Synthetic particles of known size and density were measured to calibrate the mass sensitivity of the device. Gold nanoparticles $(100 \pm 8 \mathrm{~nm})$ weighing $10 \mathrm{fg}$ more than the water they displace produced a mean frequency shift of $36 \mathrm{mHz}$ with a standard deviation of $6 \mathrm{mHz}$. On a different device, we measured a frequency shift of $310 \pm 30 \mathrm{mHz}$ for polystyrene microspheres $(1.51 \pm 0.01 \mu \mathrm{m})$ with $90.1 \mathrm{fg}$ mass excess. b, The masses of E. coli and B. subtilis in PBS were measured by passing the bacteria through the resonator and collecting peak height histograms. 
density is unknown, this value may be obtained by conducting the measurement in two different carrier solutions.

An interesting application enabled by flow-through resonant mass sensing is the weighing of individual live bacteria. The mass histograms depicted in Fig. 4 b reveal the distribution of masses within two bacterial cultures. The mass of Escherichia coli was measured to be $110 \pm 30 \mathrm{fg}$ in excess of the displaced buffer, while the mass of Bacillus subtilis is $150 \pm 40 \mathrm{fg}$. This is consistent with the distinct sizes of $E$. coli $(0.8 \times 2 \mu \mathrm{m}$ rod $)$ and B. subtilis $(1 \times 2.5 \mu \mathrm{m}$ rod $)$ and reported mass density values for bacteria. The distribution asymmetry is consistent with previously reported bacterial size distributions, and is related to the bacterial growth and division process ${ }^{25}$.

Transient particle measurements require no chemical modification of the resonator and are not affected by sensor drift or bulk density changes. Furthermore, a large number of assays can be run on the same device without requiring surface regeneration. Nanoparticle-biomolecule conjugates enable a variety of interesting protocols for highly specific and extremely sensitive detection of molecules, viruses and cells ${ }^{26,27}$. Mass sensing with resonating microchannels provides an attractive readout method for such colloidal biochemical assays, owing to its ability to measure single particle masses with high resolution using inexpensive instrumentation. At present, the low flow rate of $10-100 \mathrm{pl} \mathrm{s}^{-1}$ that is required for femtogram resolution limits throughput to 1-10 particles per second. However, using arrays of suspended microchannels could substantially increase throughput by measuring particles in parallel. Another intriguing possibility is the integration of such arrays with microfluidic systems made from elastomers, which can provide pumps and valves to deliver fluids to the detector with great precision ${ }^{28,29}$.

The size of our suspended channels is appropriate for the characterization of nanoparticles and bacteria, but too small for the passage of most eukaryotic cells. Future device generations will address this limitation, and we envisage that flow-through resonant mass sensing could ultimately be configured for counting specific cells in a fashion similar to flow cytometry. For example, CD4 cells, which are routinely counted to monitor AIDS, could be labelled with nanoparticles functionalized with CD4-specific antibodies to distinguish them from other cells present in a blood sample. This method for CD4 counting could be a cheap solution in developing countries where flow cytometry is not available or too expensive $e^{30}$. By the same principle, mass labelling would enable the specific detection of pathogens such as various bacteria, viruses and toxins.

\section{Received 12 February; accepted 6 March 2007.}

1. Thundat, T., Wachter, E. A., Sharp, S. L. \& Warmack, R. J. Detection of mercury vapor using resonating microcantilevers. Appl. Phys. Lett. 66, 1695-1697 (1995).

2. Lange, D., Hagleitner, C., Hierlemann, A., Brand, O. \& Baltes, H. Complementary metal oxide semiconductor cantilever arrays on a single chip: Mass-sensitive detection of volatile organic compounds. Anal. Chem. 74, 3084-3095 (2002).

3. Lavrik, N. V. \& Datskos, P. G. Femtogram mass detection using photothermally actuated nanomechanical resonators. Appl. Phys. Lett. 82, 2697-2699 (2003).

4. Ono, T., Li, X., Miyashita, H. \& Esashi, M. Mass sensing of adsorbed molecules in sub-picogram sample with ultrathin silicon resonator. Rev. Sci. Instrum. 74, 1240-1243 (2003).

5. Gupta, A., Akin, D. \& Bashir, R. Single virus particle mass detection using microresonators with nanoscale thickness. Appl. Phys. Lett. 84, 1976-1978 (2004).

6. Ilic, B. et al. Attogram detection using nanoelectromechanical oscillators. J. Appl. Phys. 95, 3694-3703 (2004).

7. Forsen, E. et al. Ultrasensitive mass sensor fully integrated with complementary metal-oxide-semiconductor circuitry. Appl. Phys. Lett. 87, 043507 (2005).
8. Yang, Y. T., Callegari, C., Feng, X. L., Ekinci, K. L. \& Roukes, M. L. Zeptogram-scale nanomechanical mass sensing. Nano Lett. 6, 583-586 (2006).

9. Ekinci, K. L., Yang, Y. T. \& Roukes, M. L. Ultimate limits to inertial mass sensing based upon nanoelectromechanical systems. J. Appl. Phys. 95, 2682-2689 (2004).

10. Lucklum, R. \& Hauptmann, P. Acoustic microsensors-the challenge behind microgravimetry. Anal. Bioanal. Chem. 384, 667-682 (2006).

11. Weinberg, M. S., Dube, C. E., Petrovich, A. \& Zapata, A. M. Fluid damping in resonant flexural plate wave device. J. Microelectromech. Syst. 12, 567-576 (2003).

12. Zhang, H. \& Kim, E. S. Micromachined acoustic resonant mass sensor. J. Microelectromech. Syst. 14, 699-706 (2005).

13. Braun, T. et al. Micromechanical mass sensors for biomolecular detection in a physiological environment. Phys. Rev. E 72, 031907 (2005).

14. Pang, W. et al. Femtogram mass sensing platform based on lateral extensional mode piezoelectric resonator. Appl. Phys. Lett. 88, 243503 (2006).

15. Burg, T. P. \& Manalis, S. R. Suspended microchannel resonators for biomolecular detection. Appl. Phys. Lett. 83, 2698-2700 (2003).

16. Burg, T. P. et al. Vacuum-packaged suspended microchannel resonant mass sensor for biomolecular detection. J. Microelectromech. Syst. 15, 1466-1476 (2006).

17. Q-Sense. Model D3000 specifications. 〈http://www.q-sense.com/ viewArticle.asp?ID $=31\rangle$.

18. Enoksson, P., Stemme, G. \& Stemme, E. Silicon tube structures for a fluid-density sensor. Sens. Actuators A 54, 558-562 (1996).

19. Westberg, D., Paul, O., Andersson, G. \& Baltes, H. A CMOS-compatible fluid density sensor. J. Micromech. Microeng. 7, 253-255 (1997).

20. Sarid, D. Scanning Force Microscopy: With Applications to Electric, Magnetic, and Atomic Forces (Oxford Univ. Press, USA, 1994).

21. Myszka, D. G. Analysis of small-molecule interactions using Biacore S51 technology. Anal. Biochem. 329, 316-323 (2004).

22. Angenendt, P., Glokler, J., Sobek, J., Lehrach, H. \& Cahill, D. J. Next generation of protein microarray support materials: Evaluation for protein and antibody microarray applications. J. Chromatogr. A 1009, 97-104 (2003).

23. Wu, G. H. et al. Bioassay of prostate-specific antigen (PSA) using microcantilevers. Nature Biotechnol. 19, 856-860 (2001).

24. Backmann, N. et al. A label-free immunosensor array using single-chain antibody fragments. Proc. Natl Acad. Sci. USA 102, 14587-14592 (2005).

25. Akerlund, T., Nordstrom, K. \& Bernander, R. Analysis of cell size and DNA content in exponentially growing and stationary-phase batch cultures of Escherichia coli. J. Bacteriol. 177, 6791-6797 (1995).

26. Nam, J. M., Thaxton, C. S. \& Mirkin, C. A. Nanoparticle-based bio-bar codes for the ultrasensitive detection of proteins. Science 301, 1884-1886 (2003).

27. Agrawal, A., Zhang, C. Y., Byassee, T., Tripp, R. A. \& Nie, S. M. Counting single native biomolecules and intact viruses with color-coded nanoparticles. Anal. Chem. 78, 1061-1070 (2006)

28. Thorsen, T., Maerkl, S. J. \& Quake, S. R. Microfluidic large-scale integration. Science 298, 580-584 (2002).

29. Whitesides, G. M. The origins and the future of microfluidics. Nature 442 , 368-373 (2006).

30. Yager, P. et al. Microfluidic diagnostic technologies for global public health. Nature 442, 412-418 (2006).

Supplementary Information is linked to the online version of the paper at www.nature.com/nature.

Acknowledgements We thank N. Milovic, J. Behr, M.T. Thompson and K. Van Vliet for helpful discussions, A. Mirza for substantial contributions to device fabrication, and $\mathrm{A}$. Ting for a critical review of the manuscript. We also acknowledge financial support from the National Institutes of Health (NIH) Cell Decision Process Center Grant, the Institute for Collaborative Biotechnologies from the US Army Research Office, the Air Force Office of Sponsored Research and a National Science Foundation (NSF) Small Business Innovation Research award. M.G. acknowledges support from the Natural Sciences and Engineering Research Council of Canada (NSERC) through a postdoctoral fellowship.

Author Information Reprints and permissions information is available at www.nature.com/reprints. The authors declare competing financial interests: details accompany the full-text HTML version of the paper at www.nature.com/ nature. Correspondence and requests for materials should be addressed to S.R.M. (scottm@media.mit.edu). 